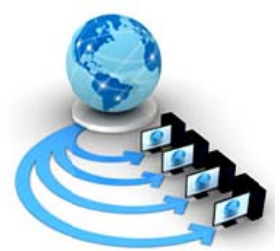

Volume 9, No. 1, January-February 2018

International Journal of Advanced Research in Computer Science

RESEARCH PAPER

\title{
CHANNEL ASSIGNMENT ALGORITHM BASED ON INTERFERENCE REDUCTION FOR MULTI-RADIO MULTI-CHANNEL WIRELESS MESH NETWORKS
}

\author{
Jatinder Singh Saini \\ Research Scholar, I.K. Gujral Punjab Technical University \\ Jalandhar, Punjab, India.
}

\author{
Balwinder Singh Sohi \\ Chandigarh Group of Colleges \\ Mohali, Punjab, India.
}

\begin{abstract}
Multi-Radio Multi-Channel Wireless Mesh Network emerged as a flexible solution for real time applications. Rapid growth in demand of high speed networks leads towards multi-radio and multi-channel concepts in network. The concept of multi-radio increases the parallelism of data transfer and enhances the network performance. Each radio works on different frequency channel. Due to availability of limited numbers of non-overlapping channels, co-channel interference exists in network and degrades the network performance. An efficient channel assignment algorithm is required to minimize co-channel interference and to increase the network capacity. In this paper a channel assignment algorithm based on interference reduction has been proposed to mitigate co-channel interference and to enhance the network performance. NS-3 based WiMesh simulation tool has been used to evaluate the effectiveness of proposed algorithm in terms of throughput, delay and packet loss. Proposed algorithm has been validated and compared with Optimal Power Control algorithm and found that proposed algorithm performs better and enhances the network performance.
\end{abstract}

Keywords: Multi-Radio Multi-Channel Wireless Mesh Network, Channel Assignment, Power Control, WiMesh, NS-3.

\section{INTRODUCTION}

Multi-Radio Multi-Channel Wireless Mesh Network (MRMC WMN) is a reliable and cost effective network standard for providing the network services in wide areas. As shown in Figure 1 MRMC WMN consists of Mesh routers, Mesh clients and Gateway nodes. Mesh routers collect and forward traffic generated by mesh clients. Gateway nodes provide the backbone connectivity to network with wired network [1].

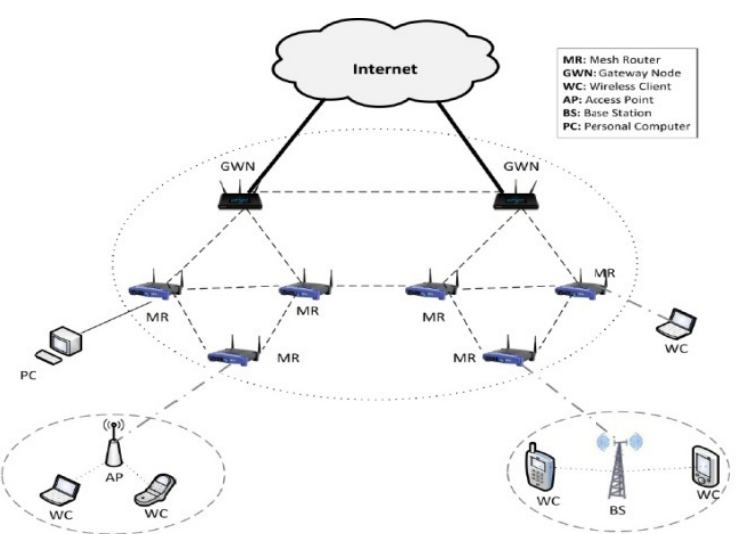

Figure. 1 MRMC WMN Architecture

With the availability of cost effective multi radio cards, it is possible to equip each mesh node with multiple radios and to achieve parallel transmission on each node [2]. Each radio is assigned with different frequency channels so that co-channel interference on each node should be minimum.

Channel assignment is a crucial issue in MRMC WMN. The process of making sure that all the interfering or neighbor nodes have been assigned with different channels is known as the Channel Assignment [3]. To ensure interference free communication in a MRMC-WMN, all the nodes within each other's communication range should ideally be on different channels. Due to availability of only few numbers of orthogonal channels, co-channel interference exists in network, which leads to limited link capacity and minimum overall network performance [4]. An efficient channel assignment algorithm is required to mitigate the problem of co-channel interference and limited link capacity. An efficient channel assignment algorithm leads towards the significant enhancement in network performance. Most of the researchers have used the fixed channel assignment approaches for channel assignment. For better network performance dynamic channel assignment algorithm based on the collective features of power control, flow control and topology control is required. A channel assignment algorithm should have important factors such as minimum connection setup time, load distribution among nodes, fault tolerance and scalability. In this paper a channel assignment algorithm based on interference reduction has been proposed to minimize the effect of co-channel interference and to increase the network capacity. Proposed channel assignment algorithm considers the important factors for channel assignment and assigns the least interference channel to link and improves the network performance. To validate the effectiveness of proposed algorithm, NS-3 based WiMesh tool has been used. WiMesh is network simulation tool and specifically used for Wireless Mesh Networks [5]. It has been developed by Stefano Avallone to carry out the research on channel assignment, power control and rate adaption algorithms. Proposed channel assignment algorithm has been compared with previous work of Optimal Power Control algorithm [6] designed using Interference Aware Topology Control channel assignment algorithm [7] in terms of throughput, delay and packet loss. Proposed algorithm is a continuous development of channel assignment algorithm as discussed in section IV. Results show that proposed algorithm performs better in comparison of Optimal Power Control algorithm (OPC). 
The rest of the paper has been structured as follows. In section II an overview of the related work has been given. In section III channel assignment problem has been analyzed. Section IV evaluates the simulation experiments for proposed channel assignment algorithm. In section $\mathrm{V}$ results are validated and compared with OPC algorithm. Finally section VI concludes the paper.

\section{RELATED WORK}

Ahmed et al. described that if two links are working on the same channel and are within interference range of each other, they will generate co-channel interference and decrease network throughput [2]. They proposed an enhanced version of Topology Controlled Interference Aware Channel Assignment algorithm based on two way interference range edge coloring model to improve the throughput ratio and fairness ratio. Yen et al. proposed a Traffic Independent, Link Centric Channel Assignment algorithm to achieve the linkpreserving requirement while minimizing overall network interference [4]. They used a Greedy Channel Assignment technique which eliminates both upper-bound and lowerbound of interference. Franklin et al. proposed Demand based State Aware Channel Reconfiguration algorithm for reconfiguration of channels [8]. This algorithm measure the traffic changes in the network and tries to reassign the channels. Algorithm minimizes the reconfiguration overhead for channel assignment and improves the network performance.

Sarasvathi and Iyengar proposed a Centralized Rank based Channel Assignment scheme, which computes the rank for each link based on the aggregate traffic, weighted cumulative expected time from gateway and number of NICs per node [9]. Aggregate traffic streaming has significant effect on the channel assignment strategy for performance enhancement. Franklin et al. described the Joint Channel Assignment and Flow Allocation problem for MRMC WMN as a Mixed Integer Linear Program [10]. They proposed the use of non overlapped and partially overlapped channel assignment to illustrate that the use of partially overlapped channels is an attractive replacement for additional non-overlapped channels. Liu and Bai depicted that network operations such as channel assignment, power control, routing and topology control are interlinked to each other [11]. Topology control can be considered as a basic operation for other network operations in MRMC WMN. Topology control plays a vital role in the routing of network data and to enhance the network performance.

Shao et al. proposed a Pure Integer Quadratic Programming based channel assignment model [12]. This model solves the static channel assignment problem in MRMC WMN by calculating the interference on each link. As compared to the existing work proposed model reduces the co-channel interfaces as well as increases the capacity of the network. Jin et al. described that in order to improve the network capacity and to improve the performance of WMN, nodes should be equipped with multiple radios and each node must be tuned on multiple non-overlapping channels [13]. Authors proposed a Link Priority-aware, Traffic Load-aware and Interferenceaware Channel Assignment algorithm. Proposed algorithm measures the level of co-channel interference and amount of traffic on each link. After this proposed algorithm then assigns the best possible channel based on their link priority and enhances the network performance.

$\mathrm{Wu}$ et al. defined that WMN achieves low throughput due to the highly unpredictable and lossy wireless channels [14]. They proposed Workload-aware Channel Assignment and Routing algorithm which takes the advantage of both opportunistic throughput gain and multi-channel throughput gain. Ye et al. proposed Flow Oriented Graph Coloring and Flow Oriented Channel Assignment algorithm to improve the channel assignment technique and network throughput [15]. Both algorithms identify bottleneck at each iteration using routing and network topology information and try to resolve the congestion. Chaudhry et al. considered topology information of each node and channel assigned to neighbor nodes to assign appropriate channel to each node. Authors proposed a Topology Controlled Interference Aware channel assignment algorithm [16], which performs better as compared to existing algorithm and decreases the co-channel interference as well as increases the network throughput.

Nezhad et al. illustrated that availability of more cost effective multi radios per node and few numbers of orthogonal channels, restricts the achievable bandwidth or link capacity on wireless links and therefore decreases the overall network performance [17]. Authors based on the utility of each link proposed a Utility based Channel Assignment algorithm which calculates the utility of each network link, sorts them in their priority order and assigns suitable channels. Kim et al. using the external co-channel interference information, proposed an Interference Aware Channel Assignment algorithm for MRMC WMN [18]. Each node uses the proposed algorithm to estimate the channel usage within the interference range and assigns channels dynamically. Galvez et al. explained that Traffic Aware Channel Assignment has gained benefit of optimizing radio resources by considering the real time traffic [19]. They purposed a Centralized Greedy Channel Assignment algorithm that supports frequent channel reassignment by using two properties i.e. low computation complexity and previous channel assignment information.

Xiang-jiao et al. proposed a Traffic based Channel Assignment algorithm for MRMC WMN based on the traffic flow between nodes [20]. This algorithm equally distributes the traffic load among each node and balances the traffic load. Proposed algorithm minimizes the network interference and enhances the network throughput. Chieochan and Hossain proposed a Centralized Auction-based Channel Assignment algorithm which assigns feasible channels for all nodes [21]. By utilizing more number of channels and by scheduling the packet transmission, unfairness of network can be managed. Based on random network coding, the proposed algorithm assigns the best channel and enhances network performance. Hao et al. proposed a Hidden Node and Interference Aware based Channel Assignment algorithm for MRMC WMN [22].This algorithm conferred that due to hidden node problem of interference, collision, transmission delay as well as packet losses may happen. They investigated correlation between the impact of radio and channel constraints. Proposed algorithm assigns channels by considering the presence of hidden nodes and reduces the interference among each node. 


\section{CHANNEL ASSIGNMENT PROBLEM}

Literature survey reveals that authors have proposed various channel assignment algorithms for MRMC WMN based on various factors such as interference aware, estimated load, power control, topology control, routing or some combinations of these. Some improvements have been shown by them in channel assignment algorithms to enhance the network performance. In these algorithms no evidence has been observed to see the comprehensive effect of all the above said factors to develop a channel assignment algorithm. Channel assignment plays a crucial role in network reliability and better network performance. So an efficient channel assignment algorithm is required, which collectively considers above said important factors for channel assignment.

In the proposed work emphasis has been given on development of an efficient channel assignment algorithm based on combination of Power Control, Topology Control and Dynamic Traffic flow Control for MRMC WMN. Proposed algorithm reduces the effect of co-channel interference and enhances the network performance.

\section{EXPERIMENT SETUP}

To validate the proposed algorithm simulation test-bed has been designed using WiMesh tool. WiMesh is a NS-3 based simulation tool used to perform experiments for channel assignment algorithms in MRMC WMN. To setup the test-bed a network of 30 nodes has been designed as shown in Figure 2.

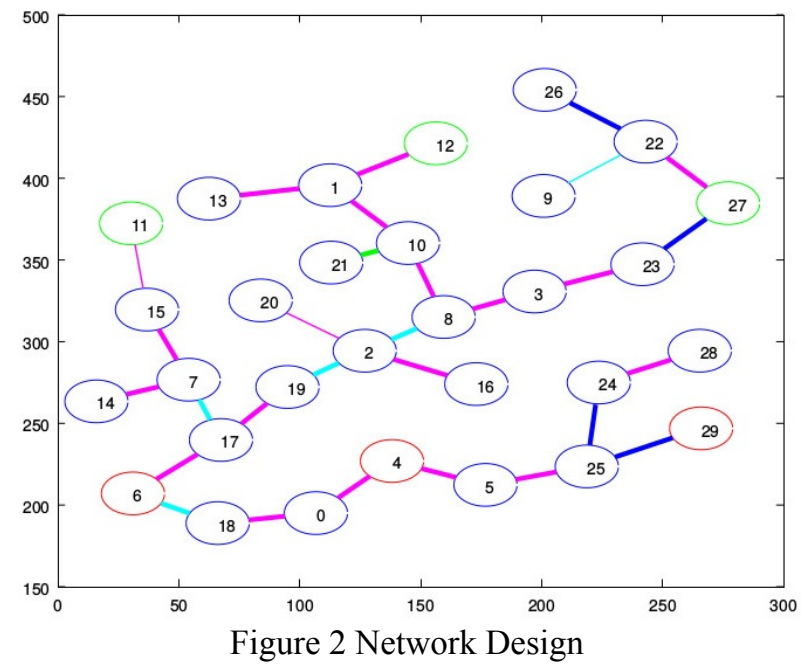

Nodes have been placed on random locations to setup the network in 300x350 area. Each node has been configured with 3 radios to implement the concept of multi radios. Network has been configured as per the parameters shown in table 1 and simulation experiment has been run for 600 seconds.

Table 1 Network Configuration

\begin{tabular}{|l|l|}
\hline Description & Parameters Value \\
\hline Simulation Time & $600 \mathrm{sec}$ \\
\hline No. of Channels & 11 \\
\hline No. of Nodes & 30 \\
\hline Power Control Algorithm & OPC Algorithm \\
\hline
\end{tabular}

\begin{tabular}{|l|l|}
\hline Traffic Type & On-Off \\
\hline Interference Model & SINR \\
\hline Transmission Speed & $6 \mathrm{Mbps}$ \\
\hline No. of radios per node & 3 \\
\hline Aggregate nodes & 3 \\
\hline Gateway Nodes & 3 \\
\hline
\end{tabular}

All available 11 radio channels have been used to setup the simulation experiment. For better network performance 3 nodes configured as gateway nodes and 3 nodes configured as aggregate nodes. Signal to interference plus noise ratio has used for interference model and On-Off traffic is used for data transmission. Transmission speed is set as 6 Mbps. Step by step working of proposed algorithm has shown in Figure 3.

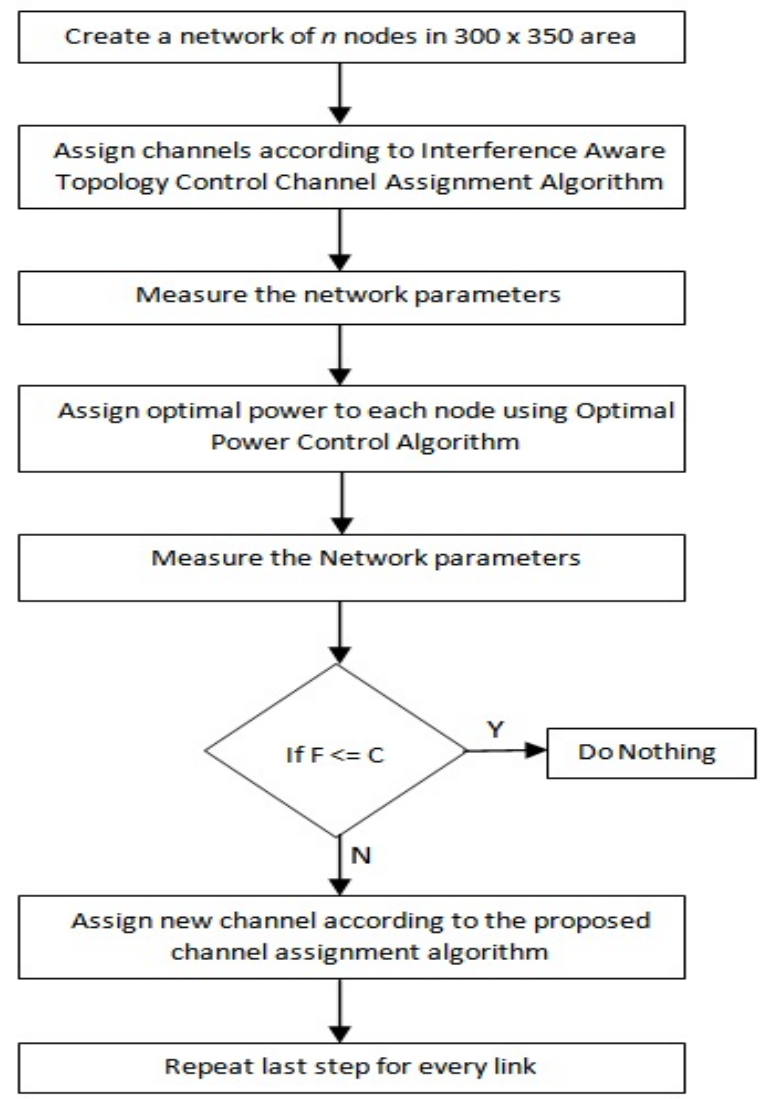

Figure 3 Work Flow

In first step network of $n$ number of nodes has been designed using simulation tool. In this work $n=30$. In second step channels have been assigned to each node by using the Interference Aware Topology Control channel assignment algorithm [7]. This algorithm ensures that each node should have minimum co-channel interference among other nodes. Then network parameters are measured in next step. In fourth step, to control the transmission power of each node Optimal Power Control algorithm has been applied. This algorithm controls the transmission power of each node up to the required transmission range and minimizes the co-channel interference. Minimum co-channel interference among network nodes ensures the better link capacity and better network performance. 
In sixth step flow $F$ and capacity $C$ is calculated and checked that, flow on link is less than and equal to the link capacity or flow is greater than the capacity. If flow is less than and equal to the link capacity then no need to change anything. If flow is more than link capacity then apply the proposed cannel assignment algorithm. Proposed algorithm reassigns the channel of concerned link, which reduces the co-channel interference and provides required link capacity. Repeat this step for every link when data transmission is initiated. New allocated channel increases the link capacity and allows more data to transfer from node to node. Thus the proposed algorithm improves the network performance in terms of throughput, delay and packet Loss.

\section{RESULTS \& DISCUSSION}

To validate the proposed, channel assignment algorithm based on interference reduction (IRCA), results have been obtained and compared with Optimal Power Control (OPC) algorithm [6]. Results have been compared in term of throughput, delay and umber of packet loss and found that proposed IRCA algorithm gave much better results.

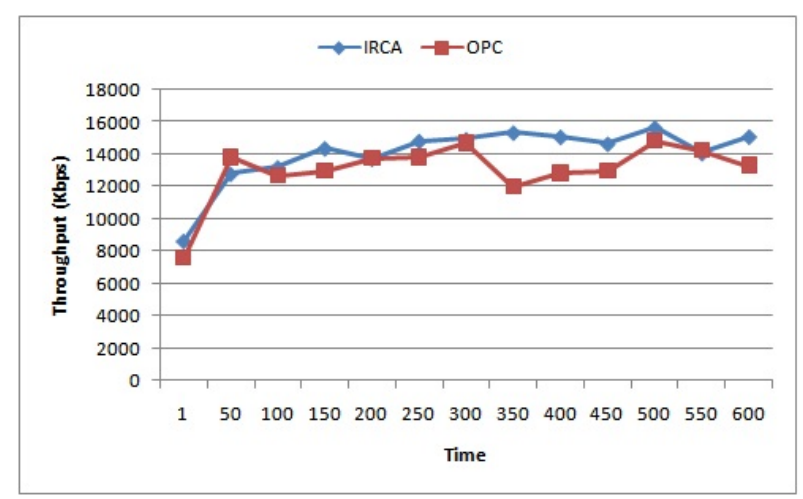

Figure 4 Throughput Comparison

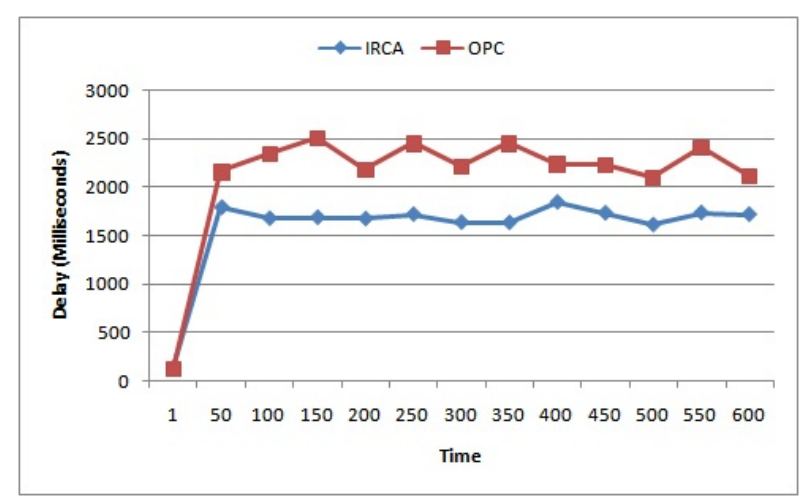

Figure 5 Delay Comparison

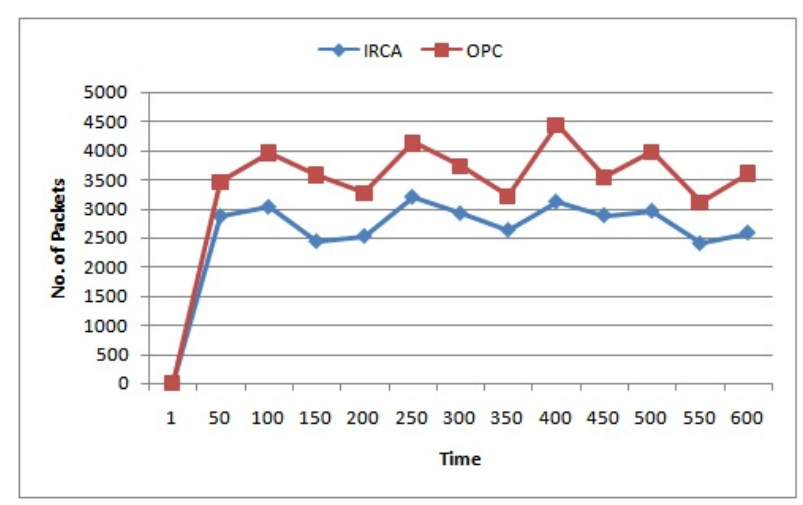

Figure 6 Packet Loss Comparisons
As shown in Figure 4 network obtains better throughput by using proposed IRCA algorithm as compared to OPC algorithm. IRCA algorithm reduces the co-channel interference and increases the link capacity, which leads towards the higher network throughput. Co-channel Interference and channel capacity are inversely proportional to each other. If co-channel interference decreases in network then link capacity improves for each link. IRCA algorithm reduces the delay in network as shown in Figure 5. IRCA algorithm provides suitable channel for each node as per demand of data flow and provides more link capacity, which allows more data to transfer at faster rate. Due to this as compared to OPC algorithm proposed IRCA algorithm exhibits less delay.

IRCA algorithm also has better result in term of number of packet loss as shown in Figure 6. Result shows that IRCA algorithm has lost less number of packets as compared to OPC algorithm. Because IRCA algorithm achieves higher throughput and more link capacity, it reduces the chances of packet lost/drop in network. Hence IRCA algorithm obtains better packet delivery ratio or drop/loss less number of packets.

\section{CONCLUSION}

Multi-Radio Multi-Channel Wireless Mesh Network (MRMC WMN) is a reliable and cost effective network standard. Multi radio concept allows to configure more than one radios per node and each radio operates on different frequency channel. Due to limited numbers of non-overlapping channels, cochannel interference exists in the network. So an efficient channel assignment algorithm is required to mitigate the effect of co-channel interference and to increase the network performance.

In this paper a channel assignment algorithm based on interference reduction has been proposed using combined features of topology control, power control and flow control. Using WiMesh simulation tool 30 nodes have been deployed on random locations to validate the proposed channel assignment algorithm. Proposed IRCA algorithm controls the transmission power of each node and assigns suitable channel, which provides sufficient link capacity as per demand of data flow. Results have been validated in terms of throughput, delay and number of packet loss and found that IRCA algorithm performs better as compared to OPC algorithm.

\section{REFERENCES}

[1] F. Bokhari, and G. Zaruba, "Partially Overlapping Channel Assignments in Wireless Mesh Networks," InTech Publisher, 2012, chap 5, pp. 103-130.

[2] N. Ahmad, A. U. Chaudhry, and R. H. M. Hafez, "Enhanced Topology-Controlled Interference-Aware Channel Assignment for Multi-Radio Multi-Channel Wireless Mesh Networks," Wireless Days, 2011, pp. 1-6.

[3] J. S. Saini, and B. S. Sohi, "A survey on channel assignment techniques of Multi-Radio Multi-Channel Wireless Mesh Network," Indian Journal of Science and Technology, vol. 9, 2016, pp. 1-8.

[4] L. Yen, K. Huang and V. Leung, "Link-Preserving Interference-Minimization Channel Assignment in MultiRadio Wireless Mesh Networks," Ad-hoc and Sensor Networking Symposium, 2012, pp. 642-647. 
[5] S. Avallone, and G. Di Stasi, "Design and implementation of WiMesh: A tool for the performance evaluation of multi-radio wireless mesh networks," Journal of Network and Computer Applications, vol. 63, 2016, pp. 98-109.

[6] J. S. Saini, and B. S. Sohi, "Optimal Power Control algorithm for Multi-Radio Multi-Channel Wireless Mesh Network," International Journal of Applied Engineering Research, vol. 13, 2018, pp. 2072-2077.

[7] J. Tang, G. Xue, and W. Zhang, "Interference-Aware Topology Control and QoS Routing in Multi-Channel Wireless Mesh Networks," 6th ACM international symposium on Mobile ad hoc networking and computing, 2005, PP. 6877.

[8] A. A. Franklin, A. Balachandran, and C.S.R. Murthy, "Online Reconfiguration of Channel Assignment in Multi-Channel Multi-Radio Wireless Mesh Networks," Computer Communications, vol. 35, 2012, pp. 2004-2013.

[9] V. Sarasvathi, and N. C. S. N. Iyengar, "Centralized Rank Based Channel Assignment for Multi -Radio Multi-Channel Wireless Mesh Networks," Computer Communication, Control and Information Technology, vol. 4, 2012, pp. 182186.

[10] A. A. Franklin, V. Bukkapatanam, and C. S. R. Murthy, "On the End-To-End Flow Allocation and Channel Assignment in Multi-Channel Multi-Radio Wireless Mesh Networks with Partially Overlapped Channels," Computer Communication, vol. 34, 2011, pp. 1858-1869.

[11] F. Liu and Y. Bai, "An Overview of Topology Control Mechanisms in Multi-Radio Multi-Channel Wireless Mesh Networks," Wireless Communications and Networking, vol. 2012, 2012, pp.1-12.

[12] B. Shao, J. Tao, and F. Wang, "Static Channel Assignment with the Physical Interference Model for Maximum Capacity in Multi-Radio Multi-Channel Wireless Mesh Networks," Grid and Cooperative Computing, 2010, pp. 338-343.

[13] D. Jin, K. Wang, and L. Feng, "PTI Channel Assignment Algorithm for Multi-Radio Multi-Channel Wireless Mesh Networks," Wireless Communications Networking and Mobile Computing, 2010, pp. 1-5.
[14] F. Wu, V. Raman, and N. Vaidya, "Being Opportunistic or Being Concurrent - On Designing Channel Assignment Algorithms in Multi-Radio, Multi-Channel Wireless Mesh Networks," SECON-2010, 2010, pp. 1-3.

[15] F. Ye, S. Roy, and Z. Niu, "Flow Oriented Channel Assignment for Multi-Radio Wireless Mesh Networks," Wireless Communications and Networking, vol. 2010, 2010, pp. 1-10.

[16] A. U. Chaudhry, N. Ahmad, and R.H. Hafez, "Improving Throughput and Fairness by Improved Channel Assignment Using Topology Control Based on Power Control for MultiRadio Multi-Channel wireless mesh networks," Wireless Communications and Networking, vol. 2012, 2012, pp. 1-25.

[17] M. A. Nezhad, L. C. Alabern, and M. G. Zapata, "Utility Based Channel Assignment: A Centralized Channel Assignment Mechanism for Multi Radio Multi Channel Wireless Mesh Networks," Mobility management and wireless access, vol. 7, 2012, pp. 68-74.

[18] Y. Kim, D. Jung, Y. Kim, S. Choi, and J. Ma, "Efficient Interference-Aware Channel Allocation in Multi-Radio Wireless Mesh Networks," Advanced Communication Technology, 2012, pp. 920-925.

[19] J. J Galvez, P. M. Ruiz, and A. F. G. Skarmeta, "TCP FlowAware Channel Re-Assignment in Multi-Radio MultiChannel Wireless Mesh Networks," Mobile Ad-Hoc and Sensor Systems, 2011, pp. 262-271.

[20] T. Xiang-jiao, H. Ting-lei, L. Xiao-yu, and L. Ming-ming, "A Channel Assignment Algorithm for Multi-Radio MultiChannel in Wireless Mesh Networks," Intelligent Computing and Integrated Systems, 2010, pp. 902-905.

[21] S. Chieochan, and E. Hossain, "Channel Assignment for Throughput Optimization in Multichannel Multiradio Wireless Mesh Networks Using Network Coding," IEEE Transactions on Mobile Computing, vol. 12, 2013, pp. 118135.

[22] F. Hao, J. Ma, C. Zhu, and L. T. Yang, "HIQP : A Hidden Node and Interference Aware Channel Assignment Scheme for Multi-Radio Multi-Channel Wireless Mesh Networks," Innovative Computing, Information and Control, vol. 8, 2012, pp. 3585-3599. 